\title{
Análisis del transporte por locomotoras eléctricas y a baterías en el nivel principal y abastecimiento de los niveles secundarios en PRODUMIN S.A.
}

Training as a generator of business value Analysis of the transport by electric locomotives and batteries in the main level and supply of the secondary levels in PRODUMIN S.A

Marco Antonio Mejía Flores. ${ }^{1}$, Karla Margot Morales Chacha. ${ }^{2} \&$ Wilmar Alejandro Ríos Cali. ${ }^{3}$

Recibido: 17-04-2020 / Revisado: 21-05-2020 / Aceptado: 15-06-2020 / Publicado: 03-07-2020

Resumen.

DOI: https://doi.org/10.33262/concienciadigital.v3i3.1268

La presente investigación establece un análisis del transporte por locomotoras eléctricas y el transporte por locomotoras a baterías en el nivel principal de abastecimiento a los niveles secundarios en la empresa minera PRODUMIN S.A., este análisis sirve para definir qué tipo de transporte es técnica y económicamente viable, así como también conocer cuáles son los factores interior mina que distorsionan al transporte logrando que sea insuficiente.

Para ello, se ha tomado en cuenta la base teórica como: resistencia a la gradiente, esfuerzo a la tracción, radio de curvatura, inclinación, entre otros. La metodología aplicada es mediante un control de tiempos de los diferentes ciclos de transporte y abastecimiento, obteniendo como resultado que el factor más relevante qué produce perdidas en el transporte por locomotoras es el descarrilamiento debido a una elevada gradiente, a la falta de potencia (HP) del motor y a la capacidad de la locomotora a batería (TM) en el abastecimiento de los niveles secundarios.

Palabras clave: Locomotoras eléctricas; locomotora a baterías; transporte minero, labores subterráneas, interior mina.

\footnotetext{
${ }^{1}$ Escuela Superior Politécnica de Chimborazo, Facultad de Recursos Naturales, Riobamba, Ecuador, email marco.mejia@espoch.edu.ec

${ }^{2}$ Escuela Superior Politécnica de Chimborazo, Facultad de Recursos Naturales, Riobamba, Ecuador, email karla.morales@espoch.edu.ec

${ }^{3}$ Escuela Superior Politécnica de Chimborazo, Facultad de Recursos Naturales, Riobamba, Ecuador, email alejandro.rios@gmail.com
} 


\begin{abstract}
.
The present investigation establishes an analysis of the transport by electric locomotives and the transport by battery-powered locomotives in the main level of supply to the secondary levels in the mining company PRODUMIN SA, this analysis serves to define what type of transport is technically and economically viable, as well as knowing what are the factors inside the mine that distort transportation, making it insufficient. For this, the theoretical basis has been taken into account such as: resistance to the gradient, tensile stress, radius of curvature, inclination, among others. The applied methodology is by means of a time control of the different transport and supply cycles, obtaining as a result that the most relevant factor that produces losses in the transport by locomotives is the derailment due to a high gradient, to the lack of power (HP) of the engine and the capacity of the battery-powered locomotive (TM) in providing secondary levels.
\end{abstract}

Keywords: Electric locomotives, battery-powered locomotive, mining transport, underground works, inside mine.

\title{
Introducción.
}

La minería subterránea existe desde el principio de la historia de la humanidad, desde entonces, ha pasado por un sinnúmero de avances tecnológicos hasta la actual mecanización de las labores mineras. (Díaz, 2006). El transporte minero constituye la operación de traslado del mineral arrancado interior mina hasta el exterior de la mina; comúnmente en la pequeña minería se utiliza el transporte discontinuo, es decir, los medios de transporte realizan un movimiento alternativo entre el punto de carga y el de descarga; generalmente en el país el más utilizado es el ferrocarril. Por consiguiente, la adquisición de los equipos suele rondar el 40-45 \% de los costos de capital de una mina $\mathrm{y}$, de ellos, un gran porcentaje se destina a las operaciones de carga, transporte y extracción. Además, solo la carga y el transporte ocupan entre un $25 \%$ y un $45 \%$ del tiempo total de un relevo normal. Por tanto, las operaciones de carga, transporte y extracción adquieren una gran relevancia en el ciclo minero, y de su rendimiento y buena organización depende en gran medida la producción (Díaz, 2006).

La Compañía Minera PRODUMIN S. A. está situado junto a la comunidad La López, Cantón Camilo Ponce Enríquez, Provincia del Azuay, actualmente la Operadora Minera viene explotando reservas mineras auríferas a través de minado subterráneo de corte y relleno convencional. Este mineral es tratado en la planta metalúrgica, que básicamente tiene dos etapas: La concentración gravimétrica y la flotación; antecediendo el proceso de conminución hasta obtener la liberación del grano. El proceso de transporte minero es medular ya que luego del arranque del mineral en el frente de explotación el traslado del material se debe realizar de manera ágil y adecuada afectando en su costo las variables de la distancia, facilidad de acceso, capacidad de carga, aporte tecnológico etc. 


\section{Metodología.}

Inicialmente se procedió a la técnica documental que se origina con la recopilación y revisión de material bibliográfico referente al tema y al sitio de interés; seguida con el levantamiento de información in situ, con recorrido de observación continuos y toma de datos en matrices elaboradas para el efecto. Con ello se permite acceder a las particularidades a ser estudiadas a fondo, con el afán de operar los datos con mayor seguridad, al soportarnos en diseños exploratorios, descriptivos y experimentales, concerniente a los dos tipos de locomotoras; finiquitando con el análisis, técnico de las características de las locomotoras sujetas a prueba frente a las características geométricas de la galería incluido las obras complementarias para la puesta en marcha con su respectivo análisis económico.

\section{Ubicación y posición geográfica}

La Operadora Minera Produmin S.A. se halla situada en el sector "La López", poblado de la cabecera cantonal Camilo Ponce Enríquez, del cantón del mismo nombre, jurisdicción de la provincia de Azuay a 4,5 km al SE de la cabecera cantonal. Circunscrita en las siguientes coordenadas:

\section{Coordenadas UTM \\ Este \\ 642993.8 \\ Norte \\ 9659075.7 \\ Cota \\ $375 \mathrm{msnm}$}

Figura 1: Mapa de Ubicación la Empresa Minera Produmin S.A.
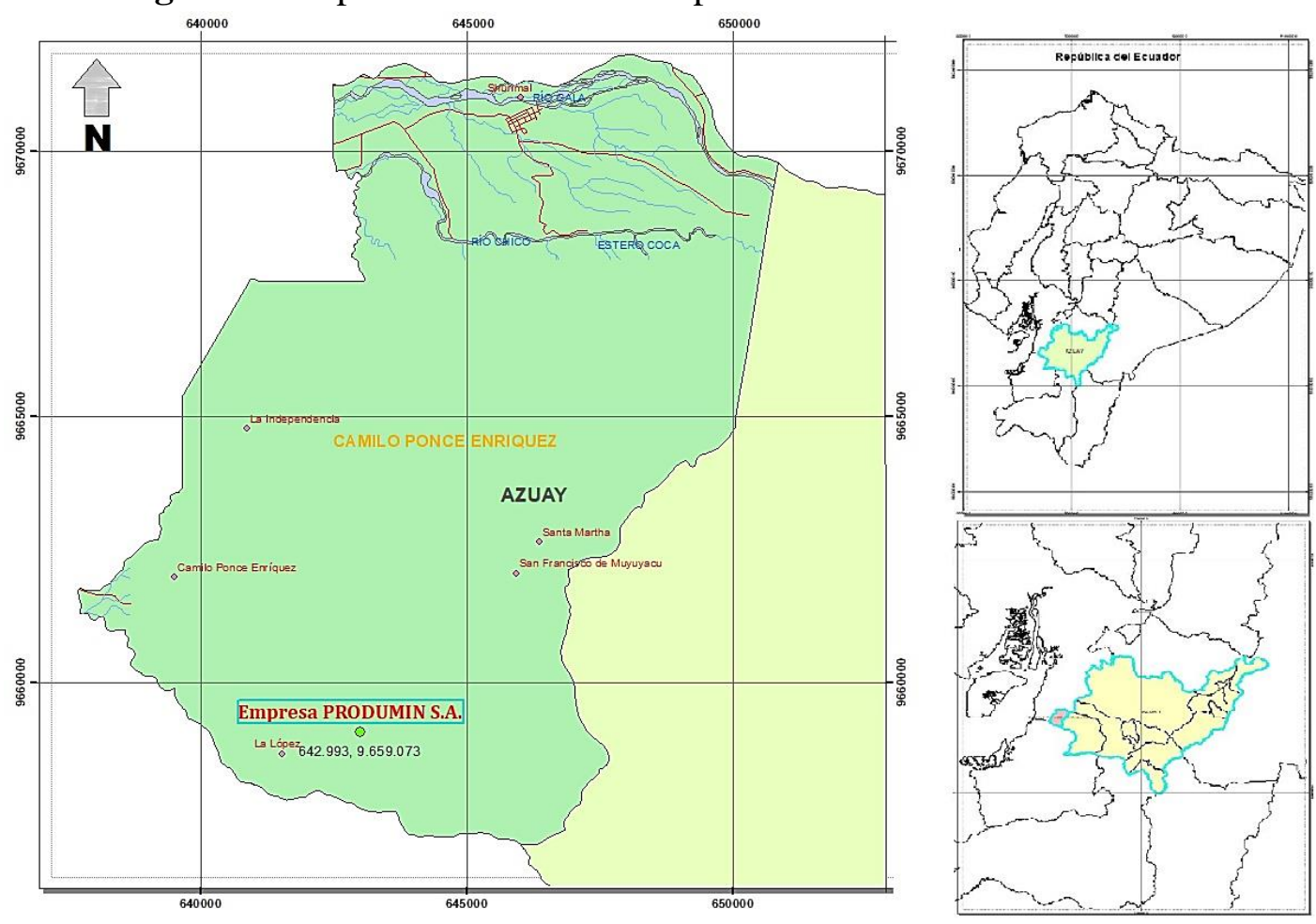

Fuente: Marco Mejía (2020) 


\section{Actividades de preparación y exploración del yacimiento}

La exploración minera es la determinación de las áreas de interés económico mediante el reconocimiento geológico y la toma de muestras de afloramiento superficial.

La preparación de la muestra es el proceso previo al análisis químico de la muestra. Este paso se considera de mucha importancia para la certeza del resultado final del análisis y consiste en las siguientes etapas: trituración, cuarteo, secado y pulverizado.

En esta etapa se emplea el muestreo para conocer las dimensiones del depósito de mineral y la distribución del mineral por su calidad y zonificación mineralógica con la finalidad de acondicionarlo para la preparación y extracción.

En este aspecto; el muestreo es más detallado y corresponde a programas de trabajo con túneles o diversos tipos de perforación, de tal forma que es posible conocer los límites del depósito, su geometría, mineralogía, saneamiento y distribución de calidad de mineral.

También se realizan diferentes tipos de labores subterráneas de preparación, las cuales permitirán llegar a las vetas, para luego continuar con las labores de explotación. En la siguiente tabla se señala las principales labores y sus dimensiones:

Tabla 1: Dimensiones de las Labores (Secciones)

\begin{tabular}{c|c|c}
\hline \multicolumn{2}{c}{ Dimensiones de las Labores } \\
\hline Labor & Secciones & Especificaciones \\
\hline Galerías & $6^{\prime} \times 7^{\prime}$ & Sobre Veta \\
Pozo(pique) & $8^{\prime} \times 10^{\prime}$ & En estéril \\
Chimenea & $4^{\prime} \times 5$, & Camino \\
Chimenea & $5^{\prime} \times 6$, & Echadero \\
Sub nivel & $4^{\prime} \times 66^{\prime}$ & Cada 35 m \\
Estocada & $4^{\prime} \times 66^{\prime}$ & Hacia los ramales/vetas \\
\hline
\end{tabular}

Fuente: Wilmar Ríos (2020)

\section{Desarrollo.}

Una vez determinado el recurso mineral y cubicado, mediante la construcción de tajos se lo extrae. Las vetas en producción actualmente son: Mary 1, Katherine 2, Kelly 3. La mineralización se encuentra emplazada en rocas ande siticas de tipo lávico masivo y almohadilladas, dentro de las cuales se formaron las vetas de tipo lazo simoide, con dirección promedio $\mathrm{N} 20^{\circ} \mathrm{W}$ y buzamiento $40^{\circ} \mathrm{E}$, las cuales son afectadas por fallas de rumbo: $\mathrm{E}-\mathrm{W}$ y NE-SW, buzamiento al $\mathrm{N}$ y NW respectivamente. Las vetas contienen minerales de: cuarzo, pirita, pirro tita, arsenopirita, calcopirita; así como también, minerales accesorios de: calcita, epidota, sericita, óxido de hierro. 
Figura 2: Veta con contenido de cuarzo, pirita, pirrotita, arsenopirita, calcopirita

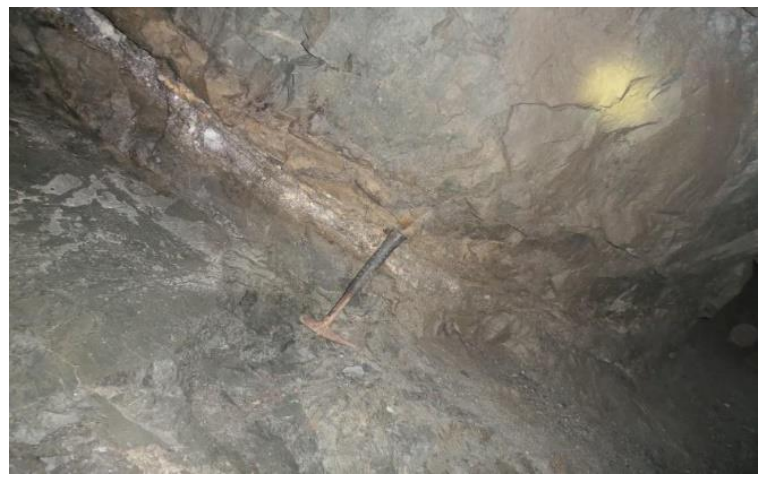

Fuente: Wilmar Ríos (2020)

\section{Actividades de preparación para la explotación}

Previamente, se comunican chimeneas extremas a una distancia de $70 \mathrm{~m}$, variando de acuerdo a los blocks de cubicación presentados por el departamento de Geología, luego en la zona media se inicia las labores de preparación con subniveles de sección 4' x 6', donde se avanza en caja generalmente al techo de la veta, para luego proceder a descolgar la veta después de comunicar a la chimenea extrema. Por lo general se deja un puente de seguridad de 3,00 $\mathrm{m}$ respecto a la galería.

\section{Métodos de Explotación estandarizado}

Corte y Relleno Ascendente Convencional. - El método utilizado es de corte y relleno ascendente convencional selectivo, es un método que garantiza una adecuada recuperación, estabilidad y selectividad del mineral. Las labores de explotación tienen en aproximadamente una longitud de $70 \mathrm{~m}$ por una altura de $50 \mathrm{~m}$ y están compuestas por uno o varios blocks.

Figura 3: Método Corte y Relleno Ascendente

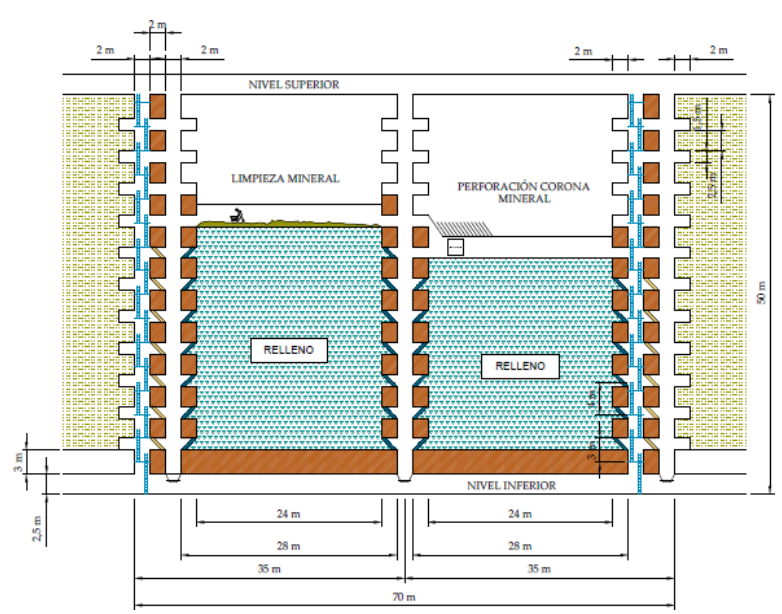

Fuente: Wilmar Ríos (2020) 
Tabla 2: Características de Diseño

\begin{tabular}{ll}
\hline \multicolumn{2}{c}{ Características de Diseño } \\
Descripción & Especificaciones \\
\hline Yacimiento & Veta angosta \\
Potencia de la veta & 0.10 a $0.15 \mathrm{~m}$. \\
& promedio \\
Buzamiento de la veta & $35^{\circ}-55^{\circ}$ \\
Forma del Tajeo & Rectangular \\
Altura del Block & $50 \mathrm{~m}$. \\
Longitud del Block & $70 \mathrm{~m}$. \\
Estribos o Pilares & $2 \times 2 \mathrm{~m}$. \\
Puente de galería a subnivel & $3.00 \mathrm{~m}$. \\
Relación desmonte - mineral & $2: 01$ \\
Recuperación en el block & $80-90 \%$ \\
Producción & $80 \mathrm{M} / \mathrm{día}$ \\
\hline
\end{tabular}

Fuente: Elaboración propia.

Las vetas por lo general son angostas (potencia de 0,10 a $0,15 \mathrm{~m}$ ), e irregulares para lo cual se emplea el "sircado", que tiene la particularidad de ser muy selectiva, consiste en disparar primero la roca caja al techo, con 2 cortes (altura 3,00 m), para luego proceder a sanear la veta.

\section{Operaciones unitarias o ciclo de minado}

Perforación. - La Perforación se realiza con perforadoras Marca Sheng Yang, empleando barrenos de 4 y 6 pies de longitud con broca de botones de $36 \mathrm{~mm}$ y $38 \mathrm{~mm}$ de diámetro. Al perforar y disparar se busca una sección en forma de arco para lograr el equilibrio de fuerzas y evitar caída de rocas.

Voladura. - Para la voladura se emplea dinamita de 1"x7" de $60 \%$ como cebo y para la columna de carga cartuchos de anfo, el diseño de la malla de voladura es con espaciamiento de 0,40 x 0,40m con un Factor de Potencia de 2,40 kg/ton.

Carguío. - Se emplea como accesorios de voladura mecha lenta y fulminantes, el proceso de carguío de taladros se realiza en función al tipo de roca, generalmente son los $3 / 4$ de la longitud del taladro. Se utiliza 1,00 m de mecha de seguridad como mecha de tiempo para el chispeo manual. Se ha establecido los horarios de disparo a las 2:30 pm. en el turno en el día y 2:30 am. en el turno de la noche.

Transporte principal por locomotoras. - Se realiza con locomotoras que tienen un motor eléctrico con batería de corriente continua, siendo la función principal la de remolcar 10 vagones. 
Figura 3: Transporte del Material con Locomotoras a Baterías

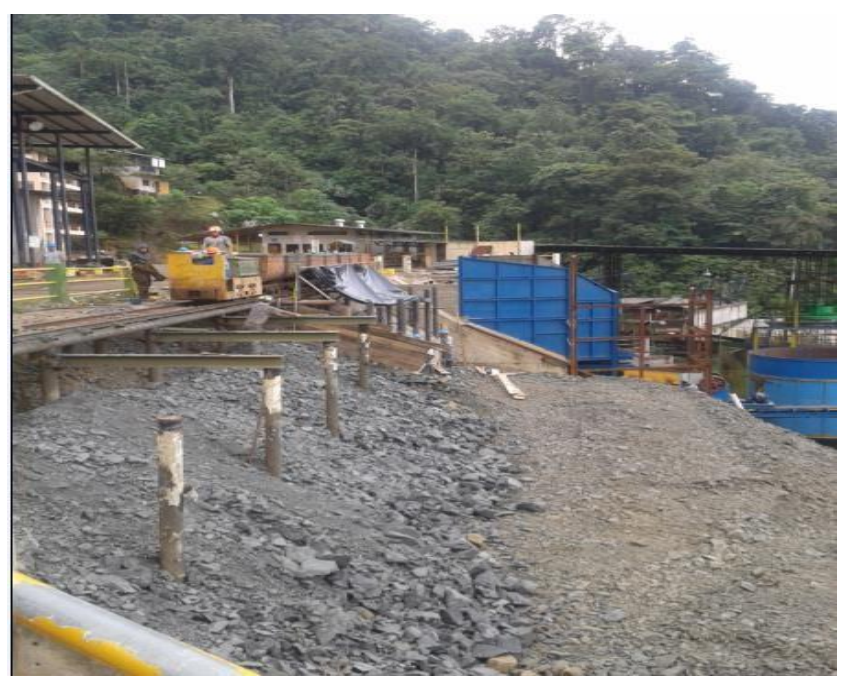

Fuente: Wilmar Ríos (2020)

Acarreo secundario (galerías y tajos). - El acarreo secundario se lo realiza desde los tajos que se encuentra en los diferentes niveles ya sean ascendentes o en los niveles inferiores. El abastecimiento (acarreo) secundario se divide en cuatro etapas:

1. Acarreo secundario con la utilización de carretillas

2. Acarreo secundario mediante carros mineros Z 20 (burras)

3. Acarreo secundario por gravedad.

4. Acarreo secundario utilizando winches de Izaje.

Figura 4: Acarreo del Material en Niveles Secundarios con Z-20.

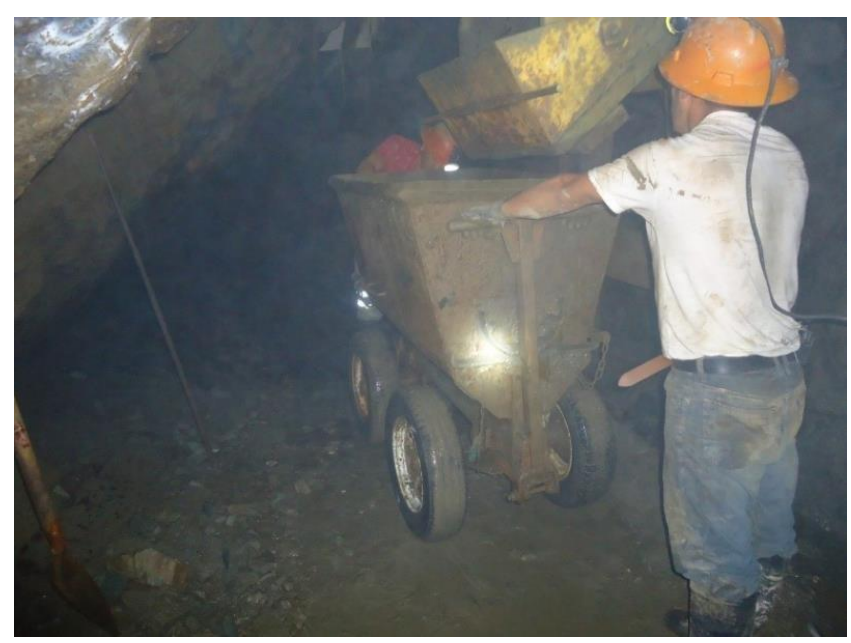

Fuente: Wilmar Ríos (2020) 


\section{Transporte.}

\section{Consideraciones generales}

El transporte comprende todas las instalaciones, mecanismos y disposiciones que sirven para mover los minerales arrancados, las tierras estériles y las máquinas, materiales y útiles necesarios, así como para el accionamiento de estas instalaciones. Los transportes interiores incluyen el movimiento entre el taller de explotación y los enganches interiores, mientras que el transporte en la superficie tiene su punto de partida en el enganche del pozo y comprende el movimiento hasta la preparación, el embarque o la escombrera.

\section{Consideraciones básicas sobre los procedimientos de transporte}

Según el tipo del proceso de transporte pueden distinguirse fundamentalmente dos grupos principales de sistemas de transporte: transportes continuos y transportes pendulares. En los transportes continuos el proceso de traslado se efectúa de una manera uniforme y en la misma dirección, mientras que en el transporte pendular el proceso se descompone en un cierto número de tramos de transporte diferentes con distinta dirección cada vez.

\section{Transportes en el interior de la mina}

La división del transporte en el interior es el resultado, en primer lugar, de sus diferentes direcciones, ya que las instalaciones de transporte varían notablemente, según se trate de trasladar masas en recorridos horizontales o aproximadamente horizontales, o en sentido ascendente o descendente, en dirección inclinada o vertical. Otra subdivisión proviene de la consideración de los lugares en los cuales se realiza el transporte, como el de las explotaciones, en las galerías de explotación, en los niveles intermedios, en las galerías principales y la extracción por pozos. Este orden corresponde al camino que siguen las zafras hacia la superficie.

Los medios de transporte que se suelen emplear en las explotaciones, valen generalmente también en las galerías de explotación y hasta la galería principal de arrastre. Por otra parte, en las galerías principales se adoptan procedimientos de transporte, que, con algunas modificaciones, están introducidos también en galerías de explotación, y que pueden ser utilizados también en explotación en capas muy echadas y con suficiente amplitud de espacio. Finalmente, la extracción por balanzas y pozos principales tienen también muchos detalles en común.

Teniendo en cuenta el empleo de los distintos procedimientos de transporte en las diferentes labores entre el frente y los enganches, resulta la siguiente distribución del transporte interior:

1. Transporte en talleres de explotación inclinados u horizontales, donde el material a transportar se mueve sobre una base. 
2. Transporte con vagonetas en galerías horizontales.

3. Transporte efectuado en dirección inclinada o vertical en balanzas, planos inclinados y pozos de extracción.

El transporte en labores inclinadas y horizontales con transportadores continuos comprende ampliamente la mayor parte del transporte en las explotaciones (tanto tajos largos como galerías, cámaras y pilares, etc.), el transporte en labores de preparación ascendentes y descendentes, como en galerías de roca, y comprende una parte del transporte en las galerías de explotación. A parte los canales fijos, se utilizan como medios de transporte los canales oscilantes, las cintas de caucho y elementos de acero y los transportadores de cadena.

También los transportes pendulares son empleados en forma de cucharas de arrastre en la explotación, especialmente en la minería de sales, y a veces también en las minas metálicas y rara vez en las hullas. El transporte sobre carriles en galerías horizontales comprende todo el transporte en las galerías principales de arrastre, el transporte en galerías de explotación, mientras no sea efectuado por transportadores continuos, y en yacimientos potentes y horizontales también el transporte en la explotación. Lo más frecuente en el transporte pendular con locomotoras, en menor escala con tractores o caballerías, o con cable abierto. Además, suele utilizarse también con un sistema de tracción sin fin con cable o cadena. Un caso especial constituye finalmente el transporte efectuado en dirección inclinada o vertical en balanzas, planos inclinados y pozos de extracción.

\section{Características de las locomotoras}

Son un tipo de maquinaria que permiten la movilización de los vagones mineros, están compuestos principalmente por motores de corriente continua, controlador de marcha, faros, interruptor, bocina, resistencia y un contacto móvil. Además, existen algunos tipos de locomotoras tales como: batería, trolley y diésel.

a. El Contacto Móvil. - Es el dispositivo que los operadores de locomotoras/ motoristas, deben tener muy en cuenta; es considerado el más importante o de mayor cuidado para evitar los accidentes con locomotoras. También se debe tener en cuenta los dispositivos que excedan las dimensiones normales de la locomotora; así, por ejemplo, los dispositivos de enganche, que también son móviles.

b. Corriente continua, para locomotoras. - El desplazamiento de las locomotoras, es por medio de dos motores de Corriente Continua (CC), que hacen girar las ruedas metálicas, para que formen la tracción en las líneas de riel. 
Figura 5: Locomotora Trolley

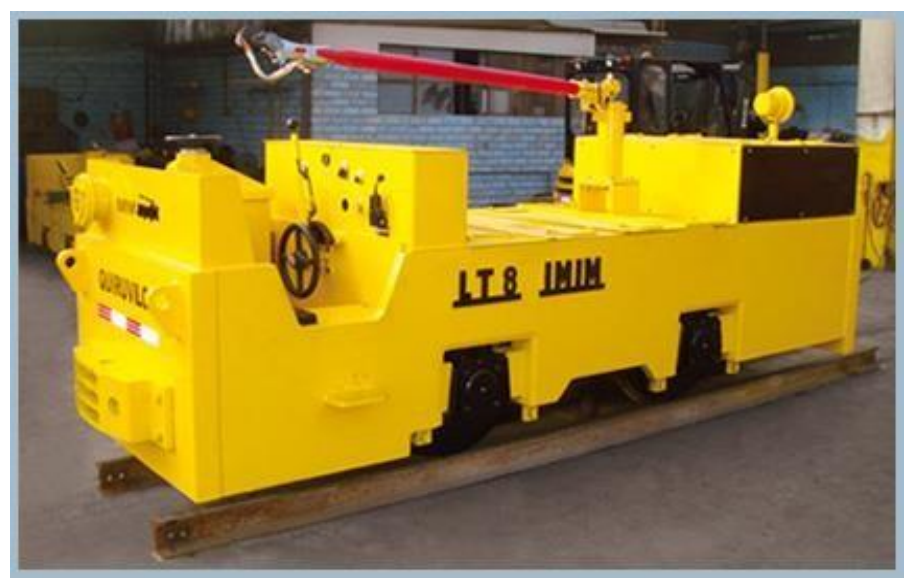

Fuente: Industrias IMIN

\section{Locomotoras acumuladoras}

Las locomotoras de este tipo funcionan mediante corriente continua, generadas ya sea por acumuladores ácidos o básicos. Su peso varía de acuerdo a su potencia, y alcanza velocidades de hasta $15 \mathrm{Km} / \mathrm{h}$. Se utilizan generalmente en minas de carbón, y en minas en donde las condiciones de trabajo y de las labores mineras no permitan de otro tipo de locomotoras.

Figura 6: Locomotora a Batería

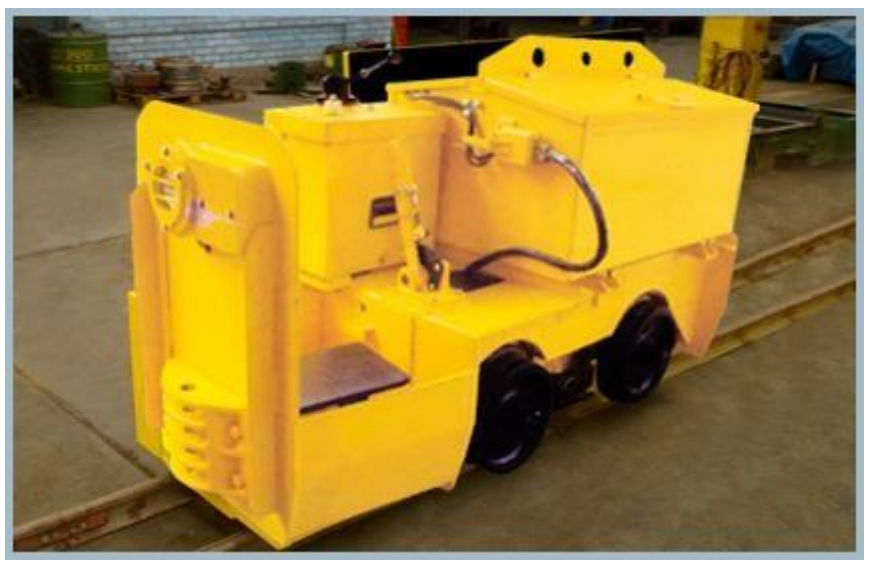

Fuente: Industrias IMIN

\section{Acumuladores o Batería}

En las minas subterráneas se utilizan locomotoras con acumuladores ácidos y alcalinos. Estos acumuladores contienen electrolito, en el cual van sumergidos unas placas paralelas. Un grupo de placas tiene la polaridad positiva (ánodo), mientras que otras 
placas intercaladas entre ellas (de composición química diferente) la negativa (cátodos). Cada grupo se conecta con un tomacorriente.

En los acumuladores ácidos; el electrolito utilizado en los acumuladores de plomo es la solución de ácido sulfúrico de 1,23 de densidad. En una batería cargada, la masa activa es el plomo esponjoso $\mathrm{Pb}$ sobre el cátodo y el peróxido de plomo $\mathrm{PbO} 2$, sobre el ánodo. La carga y descarga durante la marcha se puede representar mediante la siguiente reacción:

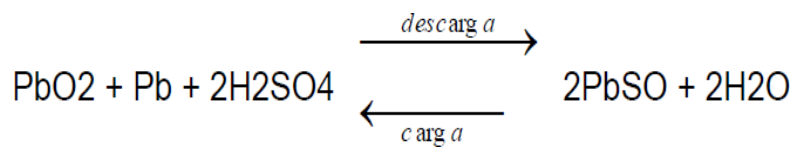

La fuerza electromotriz de un acumulador cargado es de $2.1 \mathrm{~V}$ por el elemento, decreciendo con la descarga. Al alcanzar la tensión a $1.75 \mathrm{~V}$ se debe retirar la batería del servicio, sino será destruida rápidamente por el fenómeno de sulfatación.

Los acumuladores alcalinos o básicos utilizan como electrolito la solución de potasa al $20 \%$, de una densidad 1,18 a 1,20 con agregado de $\mathrm{LiOH}$ (10g por litro). La masa activa de las placas positivas consta de una mezcla de hidróxido de níquel $\mathrm{Ni}(\mathrm{OH}) 3$ y de grafito, este último para el aumento de la conductividad; la masa activa de las placas negativas se prepara con hierro esponjoso y la reacción de carga y descarga en este tipo de acumulador es:

$$
2 \mathrm{Ni}(\mathrm{OH}) 2+\mathrm{Fe}(\mathrm{OH})+\mathrm{HOH} \underset{{ }_{\text {carg } a}}{\stackrel{\text { descarg } a}{\longrightarrow}} 2 \mathrm{Ni}(\mathrm{OH}) 3+\mathrm{Fe}+\mathrm{KOH}
$$

\section{Ventajas}

- Se puede trasportar a cualquier lugar donde exista riel

- Es muy fácil de operar

- Responde rápidamente a los controles

- No se requiere de instalación previa para la operación

\section{Desventajas}

- Se requiere de una estación de carguío de baterías

- No se puede utilizar en el trasporte de grandes tonelajes

- Tiene limitaciones de capacidad debido a la poca duración de la batería 
Figura 7: Locomotora a batería

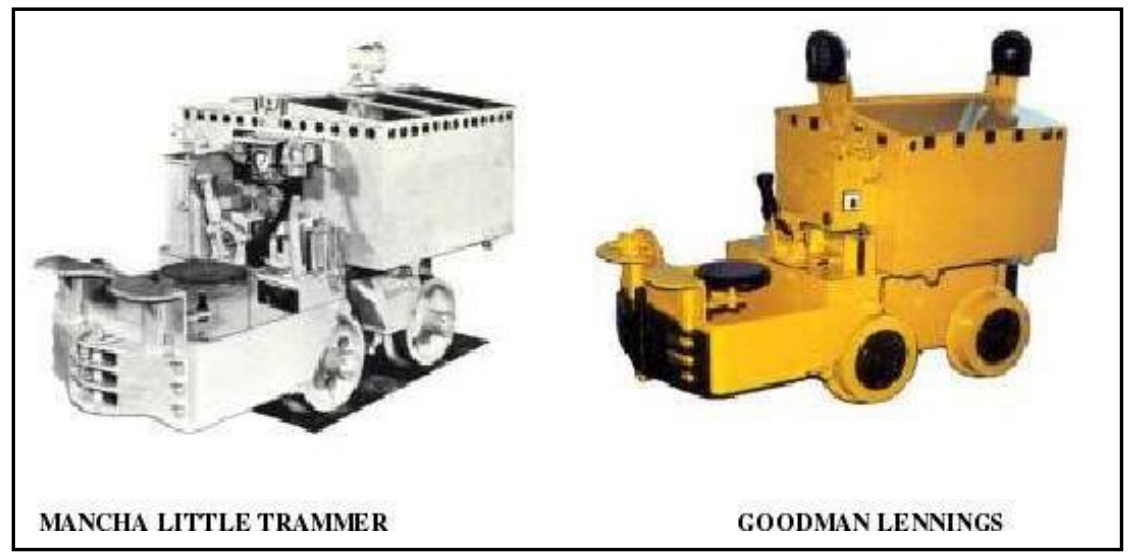

Fuente: Industrias GODMAN

\section{Resultados y análisis}

\section{Esfuerzo de tracción y potencia}

Al hacer el análisis de esfuerzo de tracción y potencia del motor para superar una gradiente de $1,7 \%$ y determinando las características del motor de las locomotoras una alternativa seria las locomotoras con Motores eléctricos por alambre trolley.

Las locomotoras eléctricas son máquinas que arrastran carros de un tren (carros mineros) y son impulsados por medio de motores que funcionan con C.C., constituidos por equipos y accesorios eléctricos y mecánicos.

Las locomotoras a trolley son las más eficientes, si bien el costo inicial es más elevado, los beneficios que reporta tener este sistema es mucho mejor. Aparte de confiabilidad es el rendimiento lo que se traduce en un menor costo/beneficio, una línea de trolley bien instalada con todos sus elementos de protección y seguridad, y con una correcta ubicación de los rectificadores a lo largo de la línea, siempre permite que las locomotoras estén alimentadas con una tensión de $+1-10 \%$ de la tensión nominal de sus motores.

\section{Características locomotoras trolley}

Las locomotoras mineras a trolley, están destinadas para el transporte horizontal sobre rieles, especialmente en minas con medio húmedo y polvoriento sin riesgo de explosión de los polvos de carbón y gases metano. Es capaz de desplazarse por carriles con pendiente superable de hasta $(2 \%)$ y a temperaturas desde -10 hasta $+35^{\circ} \mathrm{C}$. Las locomotoras en sus diferentes modelos están impulsadas por dos motores eléctricos trifásicos asíncronos con enfriamiento autónomo y con variación de revoluciones por convertidor de frecuencias, el cual facilita una marcha constante, así como también un frenado efectivo, garantizado por dos sistemas de frenos independientes para ambos 
puentes, el freno electrodinámico (con los motores eléctricos y cajas de velocidades), y los frenos mecánicos de dos circuitos directamente en las ruedas. Estas locomotoras cuentan con una o dos cabinas cerrada con visión y mando para ambas direcciones de desplazamiento.

El código de la minería da las pautas necesarias referentes a la sustentación de la línea, así como la altura mínima permisible, son las siguientes:

- La Línea de cauville o riel de $25 \mathrm{lb}$. con sus respectivos accesorios, para locomotoras de 5 TM., con carros mineros de 2 TM. Los durmientes deben ser colocados a 1 metro y con sus respectivas sapas y monas en los cambios; con guarda líneas en cambios y curvas.

- Línea trolley a un metro ochenta $(1,8 \mathrm{~m})$ de altura como mínimo desde la línea de cauville o riel.

- Establecer semáforos en cruces con su respectiva sirena.

- El circuito de la línea cauville debe ser cerrado para que la locomotora ingrese y salga jalando el convoy.

- El personal: 1 Motorista y 1 ayudante.

- La tensión de la línea trolley debe ser corriente continua mínimo de 220 V.

\section{Especificaciones de las locomotoras Trolley}

Tabla 3: Características de las locomotoras Trolley

\begin{tabular}{lccc}
\hline \multicolumn{4}{c}{ Características de la Locomotora Trolley } \\
& Locomotora & Locomotora & Locomotora \\
Características & Troley 1 & Troley 2 & Troley 3 \\
Potencia (HP) & 20 & 18 & 48.27 \\
Capacidad de & 40 & 30 & 60 \\
Arrastre (Ton) & 20 & 10 & 30 \\
Velocidad Km/H & SERMINSA WR20T & Shuangli CJY3 & Shuangli CJY6 \\
Marca & 4 & 3 & 6 \\
Peso (Ton) & $220 \mathrm{~V}$ & $220 \mathrm{~V}$ & $220 / 550 \mathrm{~V}$ \\
Fuente de Energía & &
\end{tabular}

Fuente: Elaboración propia 


\section{Análisis técnico económico}

Para el cálculo de las características del motor de las locomotoras se considera la trayectoria que tenga la mayor distancia total, es decir, la trayectoria crítica. En el caso del presente trabajo, se selecciona la segunda opción, es decir, una locomotora con motor eléctrico, por razones medioambientales (no genera monóxido de carbono, como es el caso de los motores a diésel) y requieren conexiones a fuentes de energía de forma intermitente (en el caso de trolley, se requiere una conexión constante y además existen peligros adicionales por las instalaciones de alambres que se requieren, superables con las correspondientes medidas de seguridad).

En la mina anualmente en promedio se extrae entre desmonte y mineral 132380,00 Ton. con un gasto anual de US\$161 030,66.

Tabla 4: Costos de Extracción Anual de la Mina

\section{Costos de Extracción Anual de la Mina}

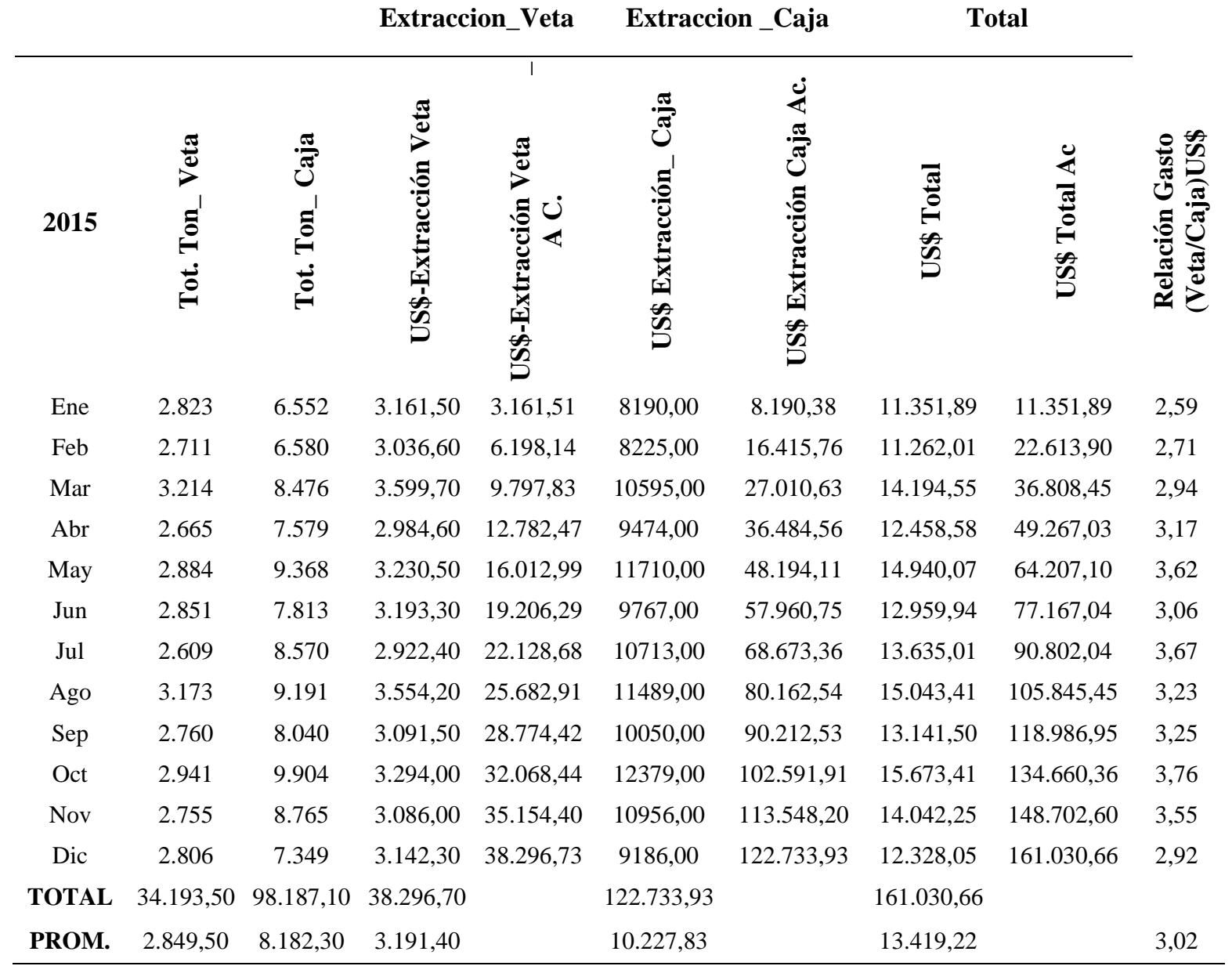

Fuente: Elaboración propia 


\section{Productividad en el transporte con locomotora}

En el gráfico mostrado obtenemos los costos unitarios por ciclo de la locomotora; de la cual, se debe hallar un "punto de equilibrio" entre el rendimiento de la locomotora y sus respectivos costos unitarios por ciclo, para determinar un punto en la cual nos indique una productividad equilibrada a un costo y distancia económica.

Esta locomotora cuenta con 10 carros U-35, el objetivo es conseguir el Rendimiento Horario (TM/h), el recorrido que realiza la locomotora en evaluación desde Bocamina a la TV $8 \mathrm{~N}$ de ciclo de trabajo $3.026 \mathrm{~km}$, transporta $13,41 \mathrm{TM} / \mathrm{h}$, a un costo de $1.46 \mathrm{US} \$ / \mathrm{TM}$ (a todo costo equipo y operación).

Al observar el grafico la productividad equilibrada a un costo y distancia económica de transporte es de $1.90 \mathrm{~km}$ y a medida que se incrementa la distancia, el tonelaje de transporte disminuye y el costo por ciclo se incrementa en $3 \%$ promedio.

Figura 8: Productividad de la Locomotora

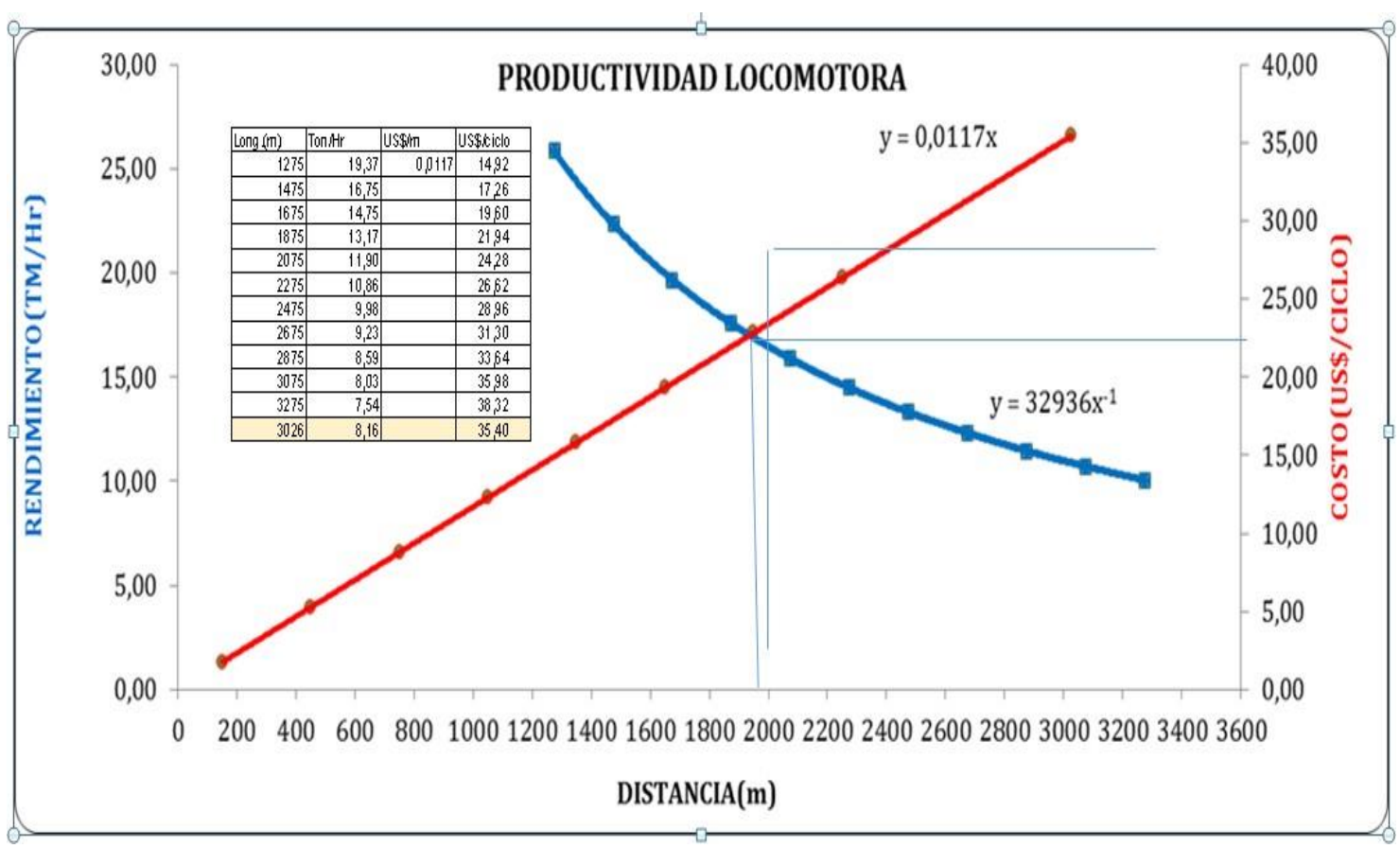

Fuente: Elaboración propia

\section{Resultados de mejoras en transporte de locomotoras}

De los gráficos mostrados podemos notar que la locomotora trolley, tendrá mayor rendimiento y menores costos, para las distancias de transporte de las locomotoras existentes, la locomotora a batería transporta $38.56 \mathrm{TM} / \mathrm{h}$, para una distancia de $3.35 \mathrm{Km}$. A un costo de 1.46 \$/TM. En cambio, la locomotora trolley rendiría $63.56 \mathrm{TM} / \mathrm{h}$, a un costo de 1.04 \$/TM, haciéndola más productiva a dicha distancia. 
Tabla 5: Cuadro de Precios de las Locomotoras

Comparativa de precios entre locomotoras a batería y trolley

\begin{tabular}{lrlr}
\hline \multicolumn{2}{c}{ Costo Locomotora a Batería } & & \multicolumn{1}{c}{ Costo Locomotora Trolley } \\
\multicolumn{1}{c}{ Descripción } & Costo anual & \multicolumn{1}{c}{ Descripción } & Costo anual \\
Costo locomotora & 5668.66 & Costo locomotora & 4400 \\
$\begin{array}{l}\text { Costo de la fuente de } \\
\text { alimentación }\end{array}$ & 9374.87 & Costo de la fuente de \\
Personal & 16200 & Personal & 4539 \\
Cargador de baterías & 11293.58 & Generador 220v/400v \\
Mantenimiento preventivo & 6034.1 & Mantenimiento preventivo & 16200 \\
Costo total anual & 48571.21 & Costo total anual & 7000 \\
Costo diario/locomotora & 133.80 & Costo diario/locomotora \\
Promedio/ton/día & $\mathbf{9 1 . 3 4}$ & Promedio/ton/día & 2400 \\
Promedio & & Promedio & 34539 \\
viajes/locomotora & $\mathbf{6 . 1}$ & viajes/locomotora \\
Costo transporte/Ton & $\mathbf{1 . 4 6}$ Costo transporte/Ton & 95.15 \\
& & & $\mathbf{9 1 . 3 4}$ \\
\hline
\end{tabular}

Observaciones: se tiene en consideración la vida útil de Observaciones: se tiene en consideración la vida útil de cada uno de los elementos como son, locomotora (5 cada uno de los elementos como son, locomotora (5 años), banco de baterías (3 años), cargador de baterías años), tendido eléctrico, generador (5años); dividiendo (5años); dividiendo el costo total para la vida útil del el costo total para la vida útil del mismo. mismo.

Fuente: Elaboración propia

\section{Análisis de los elementos para la implementación del trolley.}

Para la implementación del trolley se debe tomar en cuenta los siguientes parámetros constantes y variables para realizar la comparación entre la propuesta y las locomotoras ya existentes (baterías):

- Gradiente

- Rieles

- Tendido eléctrico

- Cambios de vía

Análisis de optimización por locomotoras a baterías: Se deben optimizar para un mayor rendimiento, la gradiente y los cambios de vía para evitar descarrilamientos. Estos parámetros deben ser mejorados ya que no son económicamente rentables, es decir, realizar un cambio de la gradiente sugiere reestructurar el diseño de la mina debido a que disminuir la gradiente de 1.7 (crítica) a 0.6 (óptima) en una distancia de 1500 metros hasta 
el lugar de carga tv $8 \mathrm{~N}$, implica cambiar 16,5 metros verticales y por un ancho de la vía de 2,5 metros lo que nos da un volumen a extraer de material estéril de 31000 metros cúbicos equivalentes a 5 meses de explotación de la mina sin beneficios.

Análisis de optimización por locomotoras trolley: De los parámetros ya mencionados para la utilización del trolley se debe implementar el tendido eléctrico que funcione con corriente continua a 220v y optimizar los cambios de vía para evitar descarrilamientos. El tendido eléctrico para el nivel principal tiene un costo de $\$ 15,00$ por metro lineal generando un costo total de 45390 dólares.

Análisis del acarreo en los niveles secundarios: Actualmente el transporte en las galerías o niveles secundarios, se lo realiza mediante carros mineros Z20 con la ayuda de dos a tres personas por la dificultad que demanda las condiciones del terreno tales como: no se lleva un control topográfico en cuanto a la gradiente, falta de estandarización en las secciones, irregularidades del piso de la galería; por lo que es necesario evaluar el sistema de transporte en dicho sector para mejorar sus condiciones y optimizar tiempos y costos.

Análisis del transporte de material por winchas: La situación actual del transporte de material por winchas como podemos apreciar en el control debido a la irregularidad de la superficie del pique ocurren descarrilamientos del balde ocasionando pérdidas en los tiempos. Se debe estandarizar el ángulo de los piques para optimizar los tiempos evitando descarrilamientos y optimizando tiempo.

\section{Conclusiones:}

- Para optimizar el transporte por locomotoras, sea a batería o trolley es necesario consideras parámetros técnicos (ratio de curvatura, peralte, ancho entre riel-riel y especificaciones técnicas del equipo) con el fin de evitar descarrilamientos y aumentar la eficiencia en el transporte; además en los niveles secundarios se debe estandarizar las pendientes ya que la irregularidad de los piques y el terreno provoca pérdidas de tiempos.

- El transporte con locomotoras a baterías tiene la particularidad de trabajar en mejores condiciones con gradientes no mayores a $0.6 \%$, pero al trabajar con una gradiente crítica de $1,7 \%$ es necesario mayores costos de mantenimiento con menor número de viajes, haciendo que la vida útil de éstas y de las baterías disminuyan considerablemente; mientras que, las locomotoras tipo trolley son más eficientes, ya que permiten trabajar eficientemente en pendientes elevadas al $2 \%$, debido a que en ellas son suministradas una carga constante de energía y por no ende, no es necesario detener el transporte para cambiar su fuente de alimentación (baterías).

- Al comparar entre las locomotoras a batería y trolley, se determina que es más eficiente la implementación de la locomotora tipo trolley ya que existe un ahorro 
de 42 ctvs por tonelada transportada, lo que equivale un ahorro de 6,26 dólares por viaje, dando un ahorro anual por transporte de 55 704,18 dólares.

- La locomotora trolley, tiene un mayor rendimiento y menores costos en comparación con la locomotora a batería, debido a que, para la distancia de transporte existente de $3,35 \mathrm{Km}$; la locomotora a batería transporta $38.56 \mathrm{TM} / \mathrm{h}$, a un costo de 1.46 \$/TM. En cambio, la locomotora trolley rinde $63.56 \mathrm{TM} / \mathrm{h}$, a un costo de 1.04 \$TM, haciéndola más productiva a dicha distancia.

- En la utilización de las locomotoras tipo trolley no es necesario cambiar los rieles debido a que se cuenta con tendido eléctrico que funciona con corriente continua a 220v y 120 amperios como ocurre en las locomotoras a batería.

\section{Referencias bibliográficas:}

Arias, S. (1998). Selección de locomotoras eléctricas para trabajo en minería. Simposio, Perú.

Baldeón, Z. (2011). Gestión en las operaciones de Transporte y acarreo para el incremento de la Productividad en CIA. Minera Condestable S.A. Pontificia Universidad Católica del Perú.

Buscetto, E. (2001). Conceptos generales, determinación de costos y control de gestión en un modelo de explotación minera. VII Congreso del Instituto internacional de costos. San Miguel de Tucumán, Argentina.

Dayan, A. R. (2015). Comportamiento geo ambiental de pasivos a partir de datos mineralógicos y análisis químicos de metales pesados de la zona Ponce EnríquezBella Rica, Provincia del Azuay, Loja.

Díaz, M. (2006). Carga, transporte y extracción en minería subterránea. Asturias, España: Septem.

Dunod, S. (1996). Trasporte, ventilación y servicios generales del fondo (Vol. II). Barcelona, España: OMEGA.

Fritzsche. C. (1988). Reglamento General de Normas Básicas se Seguridad Minera. ITC

Gallardo, A. (2003). Estudio de costos con metodología ABC de productos de la comisión chilena de energía nuclear". Universidad de Chile.

García, E. O. (2014). Optimización del sistema de explotación utilizado en la mina liga de oro. Quito, Ecuador.

Gobierno Autónomo Descentralizado del Cantón Camilo Ponce Enríquez. (2014). Plan de Desarrollo y Ordenamiento Territorial Del Cantón Camilo Ponce Enríquez. Camilo Ponce Enríquez, Ecuador.

Iglesias, J. A. (2013). perfeccionamiento del sistema de laboreo del nivel principal de la mina subterránea liga de oro, SOMILOR S.A. Cuenca, Ecuador.

Prodeminca. (2000). Evaluación de distritos mineros del Ecuador: depósitos porfídicos y Epi - Mesotermales relacionados con Intrusiones de las Cordilleras Occidental y Real. Quito, Ecuador. 


\section{PARA CITAR EL ARTÍCULO INDEXADO.}

Mejía Flores, M. A., Morales Chacha, K. M., \& Ríos Cali, W. A. (2020). Análisis del transporte por locomotoras eléctricas y a baterías en el nivel principal y abastecimiento de los niveles secundarios en PRODUMIN S.A. ConcienciaDigital, 3(3), 74-92. https://doi.org/10.33262/concienciadigital.v3i3.1268

\section{¿Ciencia}

El artículo que se publica es de exclusiva responsabilidad de los autores y no necesariamente reflejan el pensamiento de la Revista Conciencia Digital.

El artículo queda en propiedad de la revista y, por tanto, su publicación parcial y/o total en otro medio tiene que ser autorizado por el director de la Revista Conciencia Digital.
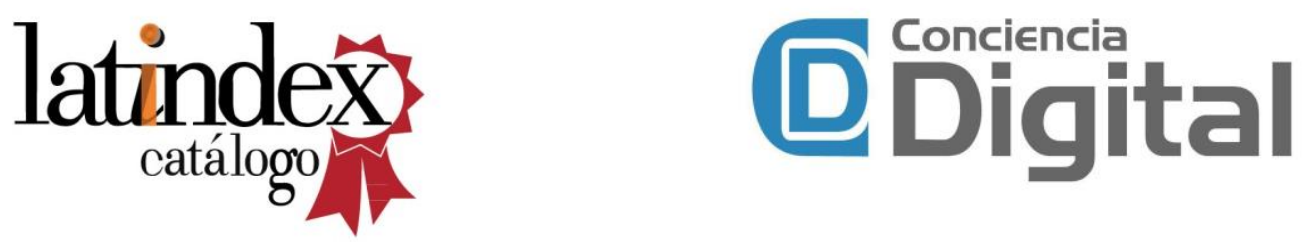